\title{
Inhibiting prepotent responses in the elderly: Distraction and disinhibition
}

\author{
Shulan Hsieh ${ }^{1,2}$ - Mengyao Wu ${ }^{1,3}$ - Chien-Hui Tang ${ }^{1}$
}

Published online: 14 September 2015

(C) Psychonomic Society, Inc. 2015

\begin{abstract}
In this study, we aimed to examine whether older adults, relative to younger adults, suffer from generic inhibition, selective inhibition, and/or distraction deficits, as assessed by behavioral and electrophysiological measures in a go/no-go task paradigm that included manipulations of nogo stimulus type (irrelevant vs. conflict) and no-go probability. A total of 96 individuals were recruited; each of three experiments included 32 participants (16 adults above and 16 adults below 60 years of age). The older adults performed more poorly than the younger adults in our behavioral test; however, the event-related potential results showed that irrelevant and conflict no-go stimuli incurred different processes that were differentially impacted by aging, as was reflected in the N2 and P3. That is, the older adults' inhibition deficits might be due to different underlying mechanisms: disproportionate processing of irrelevant no-go stimuli, and disproportionate suppression of conflicting information when executing or withholding a response to conflict no-go stimuli. The present results therefore support the theories of age-related selective inhibition and distraction deficits.
\end{abstract}

Keywords Aging $\cdot$ Inhibition $\cdot$ Distraction $\cdot$ No-go probability

Shulan Hsieh

psyhs1@mail.ncku.edu.tw

1 Cognitive Electrophysiology Laboratory: Control, Aging, Sleep, \& Emotion (CASE), Department of Psychology, National Cheng Kung University, 1 University Road, Tainan 701, Taiwan

2 Institute of Allied Health Sciences, National Cheng Kung University, Tainan, Taiwan

3 Department of Occupational Therapy, Shu-Zen Junior College of Medicine and Management, Kaohsiung, Taiwan

\section{Introduction}

As people age, many cognitive functions may deteriorate, especially the so-called cognitive control functions (Hasher \& Zacks, 1988; Kramer, Hahn, \& Gopher, 1999; Kramer, Humphrey, Larish, \& Logan, 1994; Mayr, 2001). Inhibition ability, especially, has received a great deal of attention in aging research, because inhibitory function is essential for the regulation of everyday behaviors and is of particular importance for effective adaptation in complex situations (Garavan, Ross, \& Stein, 1999). Therefore, understanding whether older adults suffer from generic deficits in inhibitory function is critical to estimate their ability to adapt to changes in situational complexity. A variety of paradigms have been employed to study inhibition (see Kok, 1999, for a review), such as the go/no-go, stop-signal, Eriksen flanker, and Stroop tasks, which are hypothesized to measure different forms of inhibitory function, such as the inhibition of a prepotent response, an ongoing response, or interference (Barkley, 1997). Several studies using these inhibition-related task paradigms have shown that older adults suffer from inhibition deficits and have further suggested that older adults suffer from generic inhibition deficits (the so-called inhibition deficit hypothesis; e.g., Dempster, 1992; Gazzaley \& D’Esposito, 2007; Hasher \& Zacks, 1988; Hasher, Zacks, \& May, 1999).

Recent studies, however, have shown that older adults do not necessarily perform more poorly than younger adults in all inhibition-related tasks, and that their performance can be affected differently by certain task parameters. For example, with a stop-signal task, older adults were found to suffer from dissociable inhibitory deficits in the sensory and motor domains (Anguera \& Gazzaley, 2012). With a go/no-go task, older adults were found to exhibit modality-specific inhibitory deficits (Falkenstein, Hoormann, \& Hohnsbein, 2002). With 
the Eriksen flanker task (Eriksen \& Eriksen, 1974), the performance of older adults in suppressing the flankers' interference was, counterintuitively, found to be better when they were required to make a noncorresponding (reversal) response to the central target (e.g., to press a left key if the central target pointed to the right) or when the central target had a salient feature (e.g., a colored central target that was easily distinguishable from the flankers; Hsieh \& Fang, 2012; Hsieh, Liang, \& Tsai, 2012; Hsieh \& Lin, 2014). Likewise, in visual search tasks, older adults were more capable of suppressing task-irrelevant information when the distracting stimuli were rare (Hommel, Li, \& Li, 2004) or were easily distinguishable from the targets on the basis of salient perceptual features (Carlson, Hasher, Zacks, \& Connelly, 1995; Scialfa, Esau, \& Joffe, 1998). Hence, older adults' inhibitory deficit might be selective, rather than generic, depending on processing domains, stimulus modalities, and/or the distinctiveness of relevant and irrelevant information (see also Kramer et al., 1994); as regards distinctiveness, the current results are inconclusive.

Although some studies have reported that the older adults can successfully suppress distinguishable irrelevant information (Carlson et al., 1995; Hsieh \& Lin, 2014; Scialfa et al., 1998), others have shown that introducing distraction to a task exacerbated age-related differences in task performance, which supports the distractibility hypothesis of aging (see Healey, Campbell, \& Hasher, 2008, for a review). Hence, aging may not only be associated with decreased inhibition ability, but also with increased susceptibility to distraction that worsens the elders' ability to identify irrelevant information. Although these two mechanisms are not exclusive, it is still important to examine how different types of irrelevant information (with different degrees of distinctiveness from relevant information) might affect elders' ability to discriminate between relevant and irrelevant information. Therefore, this study was designed to directly address this question by using a go/no-go task.

In a conventional go/no-go task, participants are instructed either to respond (go) or not to respond (no-go) to a predefined set of stimuli. In this study, two types of irrelevant information (i.e., no-go stimuli) could be manipulated: irrelevant no-go and conflict no-go stimuli. The irrelevant no-go (irNOGO) stimuli belonged to a different semantic category from that of the go stimuli (e.g., numbers vs. letters), which yielded an obvious distinction between the target (go) and nontarget (nogo) stimuli; hence, these no-go stimuli might evoke a greater bottom-up distraction effect (i.e., attentional capture), and subsequently might involve more control processes used to conquer the distraction. On the other hand, the conflict no-go (cfNOGO) stimuli shared common features with the go stimuli, but simply had different combinations of these common features; hence, these no-go stimuli might incur higher interference (due to being less distinctive from the go stimuli), and subsequently might invite the engagement of top-down control processes to resolve the conflict. By manipulating these two types of no-go stimuli, we could address the issue of whether aging increases susceptibility to distraction and/or conflict.

In addition to the type of no-go stimuli, we also manipulated the number of no-go trials in a block $(20 \%$, $50 \%$, or $80 \%$ ); our rationale for this was that, by strongly biasing the test toward go stimuli (20\% no-go probability; high-go-prepotency condition), one can maximize the engagement of executive control to inhibit no-go stimuli (Bruin \& Wijers, 2002; Ford et al., 2004), which proved successful in activating frontal lobe structures that have been associated with executive control in other response inhibition tasks (MacDonald, Cohen, Stenger, \& Carter, 2000). Accordingly, we expect that the performance of a go/no-go task might be more sensitive to aging in this higher-demand condition (e.g., 20\% of no-go probability). Nevertheless, if aging results in a generic inhibition deficit, we would likely observe an age-related inhibition deficit in the lower-demand condition as well (e.g., $80 \%$ no-go probability).

Because no overt behavioral response can be recorded for no-go trials, in this study we employed the event-related potential (ERP) method to uncover the underlying neural processes of the no-go trials. Some ERP components, such as the $\mathrm{N} 2$ and $\mathrm{P} 3$, are sensitive to inhibition and/or response conflict control processes (see Pires, Leitão, Guerrini, \& Simões, 2014, for a review). Stimuli that trigger a tendency to make incorrect prepotent responses (e.g., incongruent or no-go stimuli) were associated with enhanced fronto-central N2 amplitudes (known as the no-go N2), which is thought to reflect inhibition (Falkenstein, Hoormann, \& Hohnsbein, 1999; Roche, Garavan, Foxe, \& O'Mara, 2005; van Boxtel, van der Molen, Jennings, \& Brunia, 2001) and/or conflictmonitoring processes (Enriquez-Geppert, Konrad, Pantev, \& Huster, 2010; Huster, Enriquez-Geppert, Lavallee, Falkenstein, \& Herrmann, 2013; Nieuwenhuis, Yeung, van den Wildenberg, \& Ridderinkhof, 2003; van Veen \& Carter, 2002a, b). Hence, the functional meaning of the no-go N2 is still debated (see Smith, Johnstone, \& Barry, 2007). Following the N2, a positive ERP component peaks at approximately 250-500 ms for both go trials (known as the go P3, which usually shows maximal amplitudes at the $\mathrm{Pz}$ or $\mathrm{Cz}$ site) and no-go trials (known as the no-go P3, which usually shows maximal amplitudes at the $\mathrm{FCz}$ or $\mathrm{Cz}$ site). Both the go and no-go P3s may reflect context updating (Donchin \& Coles, 1988), which is necessary for successful ongoing execution and inhibition of prepotent responses. Relative to the no-go $\mathrm{N} 2$, the functional meaning of the no-go P3 has more consistently been shown to be closely related to inhibition (e.g., Enriquez-Geppert et al., 2010; Fallgatter \& Strik, 1999; Huster et al., 2013; Roberts, Rau, Lutzenberger, \& 
Birbaumer, 1994; Smith, Johnstone, \& Barry, 2008; TekokKilic, Shucard, \& Shucard, 2001).

\section{Hypotheses}

With the measures of these two ERP components and the behavioral data, some predictions could be derived from the existing cognitive hypotheses of aging. On the basis of the generic age-related inhibition deficit hypothesis, we would expect the older adults to exhibit slower reaction times on go trials and more errors on both go and no-go trials behaviorally, as well as smaller and delayed no-go P3 and/or no-go N2 components for both types of no-go stimuli, than younger adults across all conditions. On the basis of the selective age-related inhibition deficit hypothesis, we would expect the above-mentioned inferior performance from the elderly to be specifically associated with the cfNOGO stimuli, and such a deficit might be more prominent in the high-demand $20 \%$ no-go probability condition. On the other hand, if older adults are more susceptible to distraction, we might expect to see that older adults paradoxically exhibit larger no-go P3 amplitudes associated with the irNOGO stimuli, since older adults' attention might be captured more by the irNOGO stimuli, and therefore they must recruit more control processes to prevent responses on these no-go trials (Sawaki \& Katayama, 2008). Finally, it is also possible that the different types of nogo stimuli might incur different control processes that are differentially affected by aging.

\section{General method}

Three experiments were conducted in this study, with three different manipulations of no-go stimulus probability: $20 \%$, $50 \%$, and $80 \%$.

\section{Stimuli}

The stimuli were generated by the E-Prime software (Psychology Software Tools, Inc., Pittsburgh, PA) and were presented in red or blue against a black background at the center of a computer screen that was placed at a distance of $90 \mathrm{~cm}$ from the participant. In this task, the go stimuli were red Os and blue Xs, and the no-go stimuli were either blue Os and red Xs (designed as conflict no-go stimuli) or the red and blue numbers 3 and 6 (designed as irrelevant no-go stimuli).

\footnotetext{
${ }^{1}$ A between-subjects design for the three no-go trial probability conditions was adopted due to time constraints. Requiring individuals to participate in all three of the experiments would have been too tiring for the older participants, because each experiment required from 1 to $2 \mathrm{~h}$ to complete as well as $2 \mathrm{~h}$ of preparation for the electrophysiological recording.
}

\section{Design and procedure}

Each trial began with the presentation of a white fixation cross, "+," for a duration of $200 \mathrm{~ms}$, followed by a go or no-go stimulus for a duration of $300 \mathrm{~ms}$. The stimulus was then replaced by a black blank screen while the participant responded (or until $1,800 \mathrm{~ms}$ had elapsed, if no response was recorded), followed by an additional waiting duration that varied randomly, between 1 to $1,000 \mathrm{~ms}$, before the next trial commenced. Participants were required to respond as quickly and accurately as possible (for a go stimulus) or to withhold a response (for a no-go stimulus).

The total number of trials in an experiment was 960 . The probabilities of no-go stimuli in an experiment were 20\% (192 trials in Exp. 1), 50\% (480 trials in Exp. 2), and 80\% (768 trials in Exp. 3). Among the no-go trials, conflict no-go and irrelevant no-go stimuli comprised $50 \%$ of the trials apiece. At least 36 practice trials (more trials if necessary) were given before the formal experiment. During the practice session, "correct" or "incorrect" feedback was given at the end of each trial to facilitate the participants' familiarization with the response rule.

\section{Electroencephalographic (EEG) recording}

During the experiment, the participants were seated in a comfortable chair in a sound-attenuated room. Continuous EEG activity was recorded by 32 scalp electrodes using an electrode cap (Neuroscan Q-cap: AgCl-32 electrode cap; Neuroscan, Inc., El Paso, TX, USA). Horizontal electrooculograms (EOGs) were recorded by electrodes attached to the outer canthi of each eye. Meanwhile, vertical EOGs were recorded by two electrodes, one placed $2 \mathrm{~cm}$ below and the other $2 \mathrm{~cm}$ above the left eye. The electrodes were initially referenced online to the left mastoid and offline to the average of the left and right mastoids. The electrode impedances were maintained below $5 \mathrm{k} \Omega$. The EEG and EOG signals were amplified using SYNAMPS amplifiers. The signals were sampled at $500 \mathrm{~Hz}$ and filtered online using a high-pass filter with a cutoff frequency of $0.1 \mathrm{~Hz}$ and a low-pass filter with a cutoff frequency of $30 \mathrm{~Hz}$. Ocular artifacts associated with blinks were corrected with the ocular reduction command in the Neuroscan software (Neuroscan, Inc., El Paso, TX, USA) and were then further removed via an algorithm (Neuroscan software) that rejected any epoch if the signal was below -50 $\pm 50 \mu \mathrm{V}$, if the drift of the EEG from baseline exceeded $-50 \pm$ $50 \mu \mathrm{V}$, or if the analog-to-digital converters became saturated. The total rejection rates across the various conditions (roughly equally distributed across conditions) averaged approximately $29 \%, 31 \%$, and $29 \%$ for each experiment, respectively. 


\section{ERP analysis}

In each experiment, stimulus-locked epochs were taken from the continuous EEG signal and time-locked to the onset of the go/no-go stimulus, from $50 \mathrm{~ms}$ before to $800 \mathrm{~ms}$ after the target stimulus onset for all recording channels. For each channel, all stimulus-locked epochs were baseline corrected by obtaining the mean level of activity in the period from $50 \mathrm{~ms}$ before to $50 \mathrm{~ms}$ after target onset and then subtracting that average from the level of activity at the sample point. The $\mathrm{N} 2$ was maximal at the $\mathrm{FCz}$ site, whereas the $\mathrm{P} 3$ was maximal at the $\mathrm{Cz}$ site. Hence, we searched for the peak-to-peak amplitude at the $\mathrm{FCz}$ site during the time windows of 150 $350 \mathrm{~ms}$ (positive dip) and 250-450 ms (negative peak) after the onset of the stimulus and then computed the voltage difference between the positive dip and negative peak as the stimulus-locked N2 peak-to-peak amplitude. ${ }^{2}$ We searched for the peak latency and peak amplitude at the $\mathrm{Cz}$ site during the time window of 350-750 ms after stimulus onset and then computed the voltage difference between the peak amplitude relative to the baseline as the stimulus-locked P3 peak amplitude and the time point at which the P3 peak amplitude occurred as the $\mathrm{P} 3$ peak latency.

\section{Participants}

A total of 96 individuals were recruited through the Internet and local community advertisements; each of the three experiments included 32 participants (16 younger adults and 16 older adults). In Experiment 1 (20\% no-go probability; high go-prepotency), the 16 young adults (eight females, eight males) had a mean age of $21.75 \pm 1.07$ years (range: $20-24$ years) and an average of $15.50 \pm 0.82$ years of education; the 16 elderly adults (nine females, seven males) had a mean age of $66.5 \pm 4.23$ years (range: $60-78$ years) and an average of $13.38 \pm 2.58$ years of education. In Experiment 2 (50\% no-go probability; medium go-prepotency), the 16 young adults (ten females, six males) had a mean age of $21.13 \pm 1.36$ years (range 19-25 years) and an average of $15.06 \pm 0.77$ years of education; the 16 elderly adults (nine females, seven males) had a mean age of $66.69 \pm 5.61$ years (range: $60-77$ years) and an average of $13.38 \pm 3.24$ years of education. In Experiment 3 ( $80 \%$ no-go probability; low go-prepotency), the 16 young adults (nine females, seven males) had a mean age of $21.06 \pm$ 1.57 years (range: 19-24 years) and an average of $14.94 \pm$ 1.00 years of education; the 16 elderly adults (nine females, seven males) had a mean age of $64.81 \pm 3.95$ years (range: $61-$ 74 years) and an average of $13.19 \pm 2.86$ years of education.

\footnotetext{
${ }^{2}$ To avoid a possible waveform shift preceding the $\mathrm{N} 2$ component that might affect the measure of $\mathrm{N} 2$ amplitudes, especially for the elderly, we decided to measure the peak-to-peak amplitudes of the N2.
}

The two-way analysis of variance (ANOVA) on years of age with two between-subjects factors, Age and Experiment (i.e., no-go probability), showed a significant main effect of age (young: $21.33 \pm 1.30$ years vs. old: $66 \pm 4.58$ years, $F(1$, $90)=4,102.76, p<.01$, but no significant main effect of no-go probability and no significant interaction between age and nogo probability (all $p \mathrm{~s}>.05$ ). The two-way ANOVA on years of education showed a significant main effect of age (young: $13.31 \pm 2.82$ years vs. old: $15.17 \pm 0.87$ years $), F(1,90)=$ $17.95, p<.01$, but no significant main effect of no-go probability and no significant interaction between age and no-go probability (all $p \mathrm{~s}>.05$ ).

All participants provided their written informed consent, and the study protocol was approved by the Institutional Review Board of the National Cheng Kung University Hospital, Taiwan. All participants were paid NT \$500-\$1, 000 (US \$15-\$30) for approximately $3 \mathrm{~h}$ of participation. All participants were right-handed, were free of neurological and psychological disorders, and had normal or corrected-tonormal vision. The Mini-Mental State Examination (MMSE; Folstein, Folstein, \& McHugh, 1975) screened all participants for dementia on the basis of the following screening criteria: $25-30$ points $=$ normal; $21-24$ points $=$ mild dementia; $14-20$ points $=$ moderate dementia; and $\leq 13$ points $=$ severe dementia. In Experiment 1 (20\% no-go probability), the mean MMSE scores were $28.75 \pm 0.45$ for the younger adults and $27.06 \pm 1.53$ for the older adults. In Experiment 2 (50\% no-go probability), the mean MMSE scores were $28.13 \pm 1.09$ for the younger adults and $27.38 \pm 1.36$ for the older adults. In Experiment 3 ( $80 \%$ no-go probability), the mean MMSE scores were $28.63 \pm 0.50$ for the younger adults and $26.63 \pm$ 1.09 for the older adults.

The two-way ANOVA on MMSE scores showed a significant main effect of age (young: $28.50 \pm 0.76$ vs. old: $27.02 \pm$ 1.33), $F(1,90)=45.04, p<.01$, but no significant main effect of no-go probability and no significant interaction between age and no-go probability (all $p \mathrm{~s}>.05$ ).

\section{Results}

\section{Behavioral data analysis}

The first trial of each block, trials with reaction times (RTs) faster than $150 \mathrm{~ms}$, and trials with reaction times slower than $1,500 \mathrm{~ms}$ were discarded from further analysis. The behavioral results are shown in Table 1. The percentages of errors were labeled as commission errors for no-go trials and omission errors for go trials. The results of the ANOVAs on all of the behavioral and ERP data are summarized in Table 2.

RTs on go trials A two-way ANOVA with two betweensubjects factors (Age and No-Go Probability) on the RTs of 
Table 1 Behavioral data: Go reaction times (standard errors) and percentages of errors (standard errors) as a no-go probability, go/no-go stimulus type, and age group

\begin{tabular}{|c|c|c|c|c|c|c|c|c|c|c|c|c|}
\hline \multirow[b]{3}{*}{ Go reaction time (ms) } & \multicolumn{4}{|c|}{ No-Go $20 \%$} & \multicolumn{4}{|c|}{ No-Go $50 \%$} & \multicolumn{4}{|c|}{ No-Go $80 \%$} \\
\hline & \multicolumn{2}{|c|}{ Younger } & \multicolumn{2}{|l|}{ Older } & \multicolumn{2}{|c|}{ Younger } & \multicolumn{2}{|l|}{ Older } & \multicolumn{2}{|c|}{ Younger } & \multicolumn{2}{|l|}{ Older } \\
\hline & 451.0 & $(14.4)$ & 501.7 & $(14.2)$ & 516.6 & $(13.3)$ & 534.7 & $(15.1)$ & 554.5 & $(30.4)$ & 645.8 & $(16.1)$ \\
\hline Go omission error (\%) & 2.7 & $(1.1)$ & 4.1 & $(1.0)$ & 3.1 & $(0.8)$ & 10.1 & $(1.7)$ & 4.8 & $(1.7)$ & 10.7 & $(2.6)$ \\
\hline irNOGO commission error $(\%)$ & 1.7 & $(0.6)$ & 5.9 & $(1.2)$ & 1.2 & $(0.6)$ & 3.4 & $(0.9)$ & 0.6 & $(0.3)$ & 3.1 & $(1.2)$ \\
\hline cfNOGO commission error $(\%)$ & 17.2 & $(3.4)$ & 25.1 & $(2.5)$ & 9.7 & $(2.1)$ & 17.1 & $(2.0)$ & 3.9 & $(2.0)$ & 6.4 & $(1.3)$ \\
\hline
\end{tabular}

go trials showed that younger adults generally performed faster than the older adults, that RTs were faster in the $20 \%$ no-go probability condition than in the $50 \%$ no-go probability condition, and that RTs in the latter condition were faster than RTs in the $80 \%$ no-go probability condition (all Tukey tests: $p \mathrm{~s}<.01)$. We found no significant interaction between the two factors.

Percentage of errors (PEs) A repeated measures three-way ANOVA with two between-subjects factors (Age and No-Go Probability) and one within-subjects factor (Stimulus Type: go, irNOGO, cfNOGO) on PEs showed that younger adults generally performed better (fewer errors) than the older adults, and that errors were more frequent in the $20 \%$ no-go probability condition than in the $80 \%$ no-go probability condition (Tukey test on 20\% vs. 80\%: $p<.01$ ). We also observed a significant main effect of stimulus type, showing that irNOGO stimuli elicited fewer errors than go stimuli, which elicited fewer errors than cfNOGO stimuli did (all Tukey tests: $p$ s
$<.05)$. A significant two-way interaction between no-go probability and stimulus type was also apparent. A simple main effect test on this interaction showed that the effect of no-go probability was significant only on go trials $(20 \%<80 \%$ nogo probability condition; Tukey test: $p<.05)$ and cfNOGO trials $(20 \%>50 \%>80 \%$ no-go probability condition; all Tukey tests: $p s<.01)$.

Summary of behavioral data To summarize, the older adults appeared to perform more poorly than the younger adults, including having slower go RTs and more omission and commission errors; yet this age effect was not modulated by no-go probability or stimulus type (Table 2 ). In addition, the $20 \%$ no-go probability condition elicited more errors than the $80 \%$ no-go probability condition, which occurred only in the go and cfNOGO trials. Finally, withholding responses seemed to be more difficult for the cfNOGO than for the irNOGO stimuli, as reflected by the commission errors for both age groups.

Table 2 Summary results of the ANOVA and simple main effect tests for the behavioral and ERP analyses

\begin{tabular}{|c|c|c|c|c|c|c|c|c|c|c|c|c|c|}
\hline \multirow{2}{*}{$\begin{array}{l}\text { ANOVA: Age } \times \text { No-Go Probability } \times \text { Stimulus Type } \\
\text { Age }\end{array}$} & \multirow[b]{2}{*}{$F(1,90)$} & \multirow{2}{*}{$\begin{array}{l}\text { RT } \\
12.82\end{array}$} & \multirow[b]{2}{*}{$* *$} & \multicolumn{2}{|c|}{$\begin{array}{l}\text { Percentage } \\
\text { of Errors }\end{array}$} & \multicolumn{2}{|c|}{ N2 Latency } & \multicolumn{2}{|c|}{$\begin{array}{l}\text { N2 } \\
\text { Amplitude }\end{array}$} & \multicolumn{2}{|c|}{ P3 Latency } & \multicolumn{2}{|c|}{$\begin{array}{l}\text { P3 } \\
\text { Amplitude }\end{array}$} \\
\hline & & & & 19.68 & $* *$ & 16.16 & $* *$ & 8.53 & $* *$ & 1.82 & $(.18)$ & 0.62 & $(.43)$ \\
\hline No-Go Probability & $F(2,90)$ & 23.34 & $* *$ & 6.45 & $* *$ & 0.27 & $(.76)$ & 4.54 & $*$ & 2.02 & $(.14)$ & 1.64 & $(.22)$ \\
\hline Age $\times$ No-Go Probability & $F(2,90)$ & 2.02 & $(.14)^{\mathrm{a}}$ & 0.28 & $(.76)$ & 0.56 & $(.58)$ & 1.95 & $(.19)$ & 2.95 & $(.06)$ & 2.51 & $(.09)$ \\
\hline Stimulus Type & $F(1,90)$ & - & - & 75.49 & $* *$ & 17.80 & $* *$ & 42.49 & $* *$ & 24.54 & $* *$ & 37.36 & $* *$ \\
\hline Age $\times$ Stimulus Type & $F(1,90)$ & - & - & 1.41 & $(.26)$ & 1.46 & $(.24)$ & 5.18 & $* *$ & 10.39 & $* *$ & 8.57 & $* *$ \\
\hline \multicolumn{14}{|l|}{ Simple main effect of age ${ }^{b}$} \\
\hline On go trials & $F(1,270)$ & - & - & - & - & - & - & 9.48 & $* *$ & 0.37 & $(.54)$ & 0.16 & $(.69)$ \\
\hline On irNOGO trials & $F(1,270)$ & - & - & - & - & - & - & 0.82 & $(.37)$ & 15.00 & $* *$ & 6.02 & $*$ \\
\hline On cfNOGO trials & $F(1,270)$ & - & - & - & - & - & - & 12.69 & $* *$ & 0.01 & $(.93)$ & 0.01 & $(.94)$ \\
\hline No-Go Probability $\times$ Stimulus Type & $F(2,90)$ & - & - & 23.45 & $* *$ & 0.57 & $(.69)$ & 13.03 & $* *$ & 1.88 & $(.12)$ & 23.76 & $* *$ \\
\hline \multicolumn{14}{|l|}{ Simple main effect of no-go probability } \\
\hline On go trials & $F(2,270)$ & - & - & 3.24 & $*$ & - & - & 1.83 & $(.19)$ & - & - & 2.78 & $(.06)$ \\
\hline On irNOGO trials & $F(2,270)$ & - & - & 0.67 & $(.51)$ & - & - & 13.77 & $* *$ & - & - & 10.43 & $* *$ \\
\hline On cfNOGO trials & $F(2,270)$ & - & - & 40.62 & $* *$ & - & - & 4.60 & $*$ & - & - & 3.72 & $*$ \\
\hline Age $\times$ No-Go Probability $\times$ Stimulus Type & $F(2,90)$ & - & - & 1.73 & $(.15)$ & 0.62 & $(.65)$ & 1.29 & $(.31)$ & 1.07 & $(.39)$ & 1.39 & $(.29)$ \\
\hline
\end{tabular}

${ }^{a}$ Values in parenthesis following $F$ values indicate the $p$ value when statistical significance was not reached. ${ }^{\mathrm{b}}$ Results of simple main effect tests are presented in italic type. ${ }^{*} p<.05 ; * * p<.01$. 


\section{ERP data}

ERPs associated with each condition and age group at the $\mathrm{FCz}$ and $\mathrm{Cz}$ sites, as well as topographic maps, are shown in Fig. 1 (including go and irNOGO trials and the respective topographic maps) and Fig. 2 (including go and cfNOGO trials and the respective topographic maps). The repeated measures three-way ANOVAs with two between-subjects factors (Age and No-Go Probability) and one within-subjects factor (Stimulus Type: go, irNOGO, cfNOGO) were calculated for the $\mathrm{N} 2$ and $\mathrm{P} 3$, respectively.

\section{N2}

N2 peak latency The ANOVA on the N2 peak latencies showed that younger adults exhibited earlier N2 peak latencies than did the older adults, and that the N2 peak latency occurred earlier for both go and irNOGO trials than for cfNOGO trials.
N2 peak amplitude The ANOVA on the N2 peak amplitudes showed that they were larger for younger adults than for older adults and were larger in the $20 \%$ no-go probability condition than in the 50\% no-go probability condition (Turkey test: $p<$ $.05)$. There was also a significant main effect of stimulus type, showing that cfNOGO stimuli elicited the largest N2 amplitudes, whereas the go stimuli elicited the smallest N2 amplitudes (all Tukey tests: $p$ s $<.01$ ).

The simple main effect tests on the interaction between age and stimulus type showed that younger adults exhibited larger $\mathrm{N} 2$ amplitudes than did older adults on go $[F(1,270)=9.48, p$ $<.01]$ and cfNOGO stimuli, but not on irNOGO stimuli. In addition, the simple effect tests also showed that both younger and older adults exhibited larger N2 amplitudes for cfNOGO than for go stimuli (and also than for irNOGO stimuli among younger adults) (all Tukey tests: $p$ s $<.01$ ).

The simple main effect tests on the interaction between nogo probability and stimulus type showed that, for the irNOGO and cfNOGO stimuli, the amplitudes were larger in the $20 \%$ than in the $50 \%$ and $80 \%$ no-go probability conditions

(a) Raw ERP
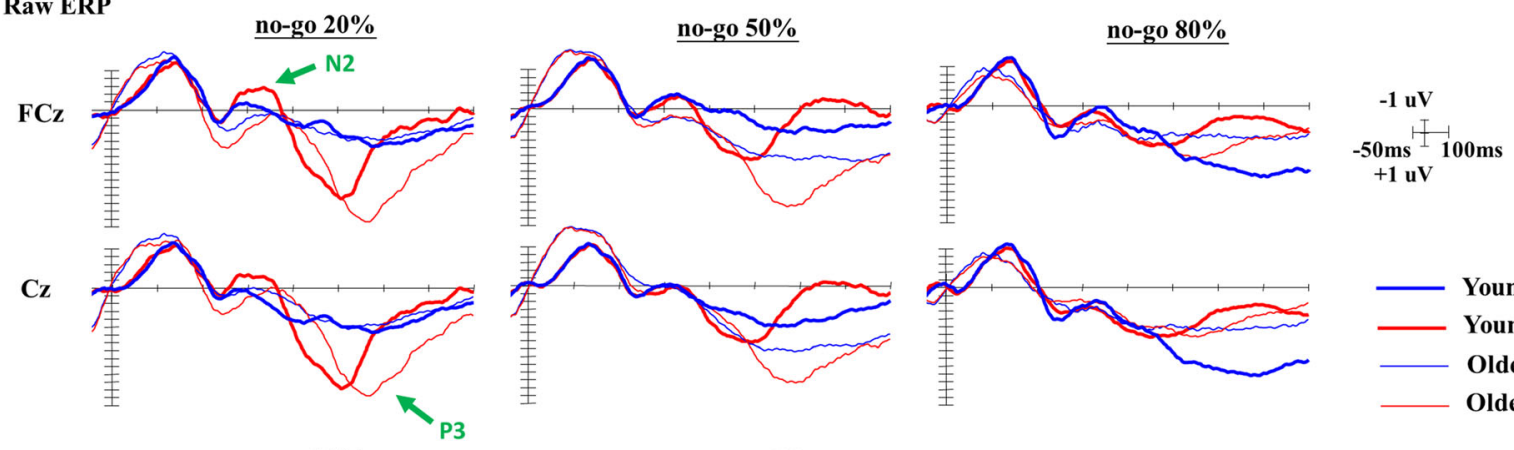

(b) $\mathrm{N} 2$
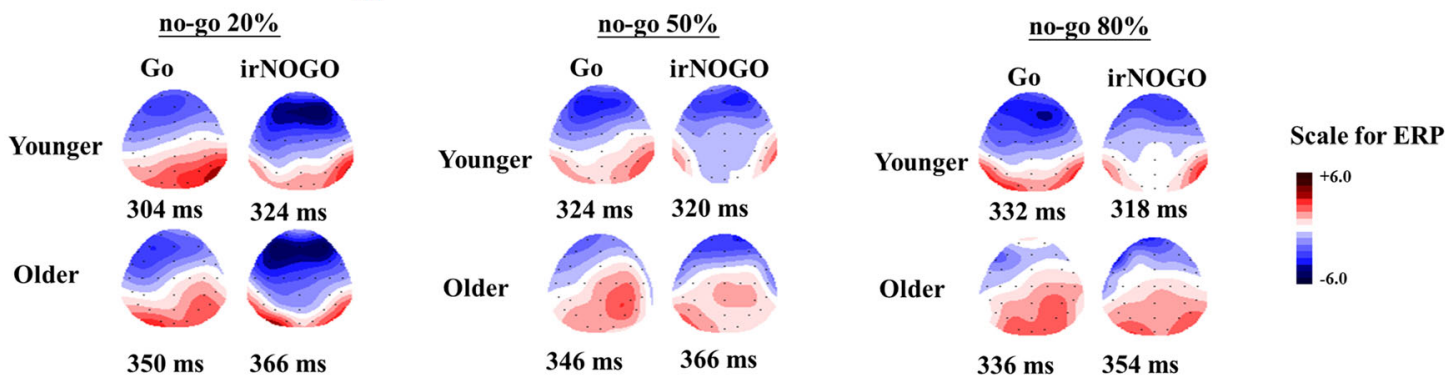

(c) P3
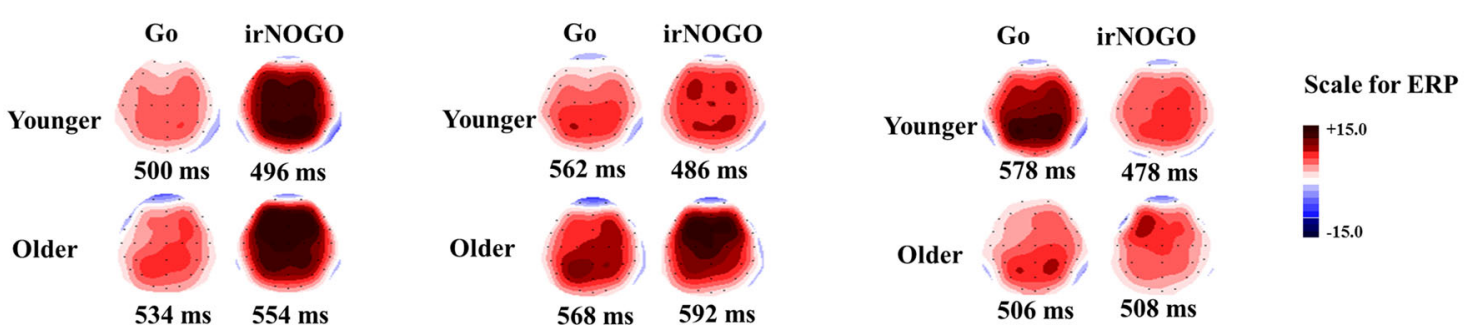

Fig. 1 (a) Event-related potential (ERP) waveforms for go and irNOGO trials in the $20 \%, 50 \%$, and $80 \%$ no-go probability conditions, separately at the $\mathrm{FCz}$ and $\mathrm{Cz}$ electrode sites for younger and older adults. (b) Topographic maps of the go N2 and irNOGO N2, separately for each no-go

stimulus probability and both age groups. (c) Topographic maps of the go P3 and irNOGO P3, separately for each no-go stimulus probability and both age groups. Each map describes the topographic distribution at the indicated peak latency for each condition 
(a) Raw ERP
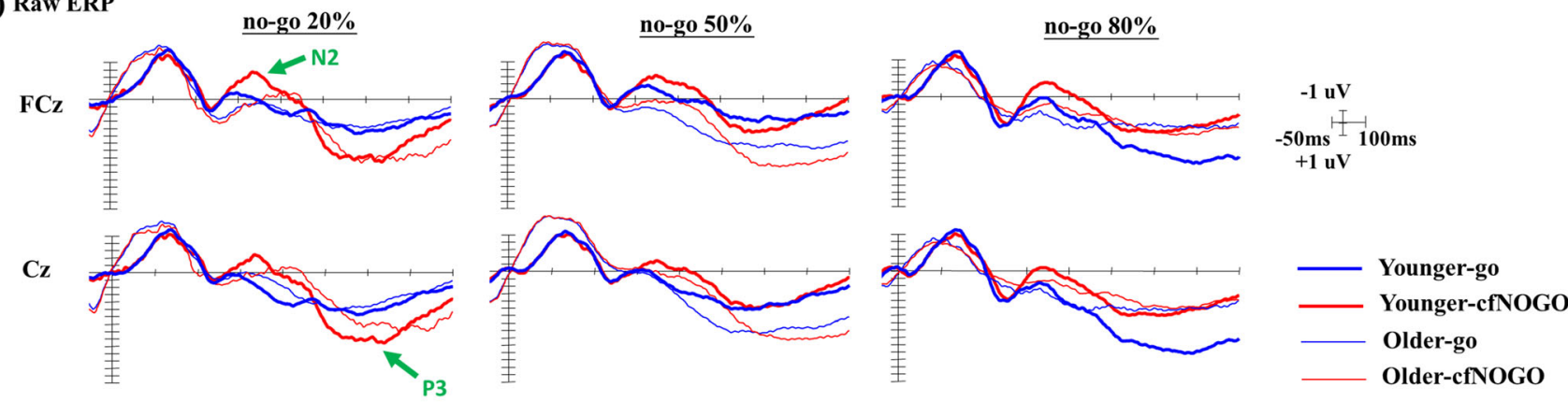

(b) $\mathrm{N} 2$
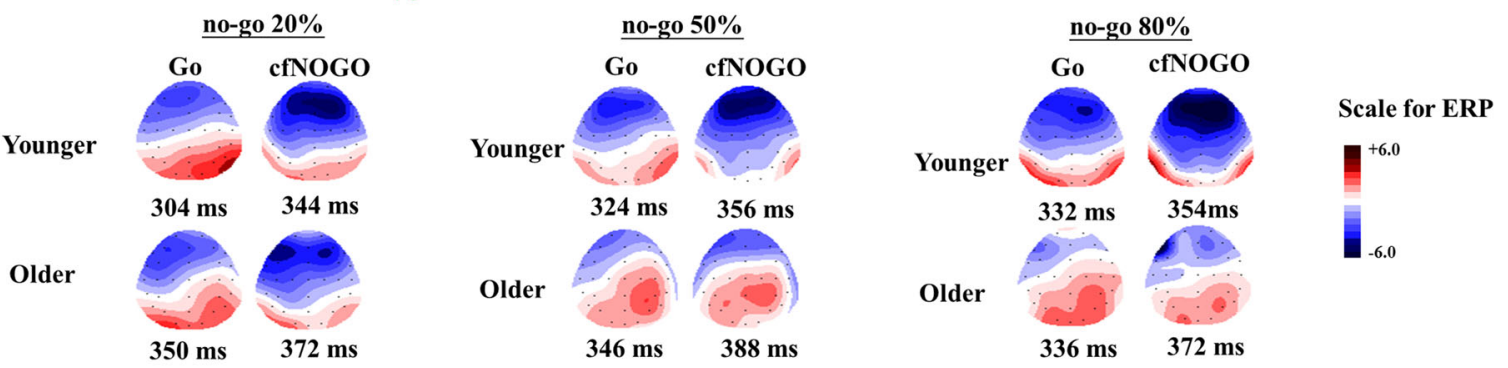

(c) P3
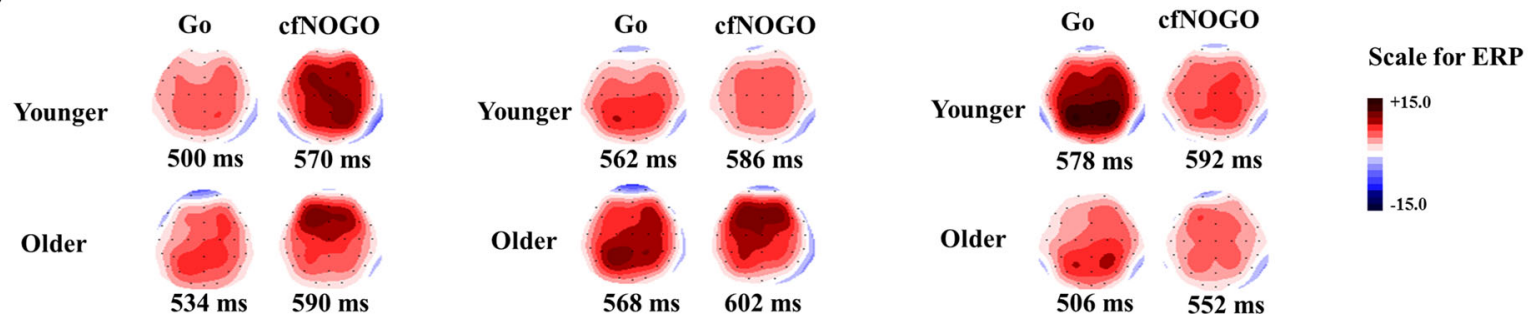

Fig. 2 (a) Event-related potential (ERP) waveforms for go and cfNOGO trials in the $20 \%, 50 \%$, and $80 \%$ no-go probability conditions, separately at the $\mathrm{FCz}$ and $\mathrm{Cz}$ electrode sites for the younger and older adults. (b) Topographic maps of the go N2 and cfNOGO N2, separately for each no-

go stimulus probability and both age groups. (c) Topographic maps of the go P3 and cfNOGO P3, separately for each no-go stimulus probability and both age groups. Each map describes the topographic distribution at the indicated peak latency for each condition

(irNOGO: $20 \%>50 \%$ \& 80\%, Tukey tests: $p \mathrm{~s}<.01$; cfNOGO: $20 \%>50 \%$; Tukey tests: $p \mathrm{~s}<.01$ ).

Summary of N2 data To summarize, older adults had longer $\mathrm{N} 2$ peak latencies and smaller N2 amplitudes than younger adults for both go and cfNOGO stimuli, but not for irNOGO stimuli (Table 2). In addition, both age groups exhibited larger $\mathrm{N} 2$ amplitudes for cfNOGO than for go stimuli (and also than for irNOGO stimuli among younger adults). For both no-go stimulus types, the $\mathrm{N} 2$ amplitudes were modulated by no-go probability, with larger N2 amplitudes being associated more with the $20 \%$ no-go probability than with the other no-go probability conditions.

\section{P3}

P3 peak latency The ANOVA on the P3 peak latencies showed the latest P3 peak latency on cfNOGO stimuli and the earliest P3 peak latency on irNOGO stimuli (Tukey test: $p \mathrm{~s}<.05)$. We also found a significant two-way interaction between age and stimulus type. The simple main effect tests on this interaction showed that younger adults exhibited earlier P3 peak latencies than older adults only for irNOGO stimuli, but not for go and cfNOGO stimuli.

P3 peak amplitude The ANOVA on P3 peak amplitudes showed larger P3 amplitudes for irNOGO than for go and cfNOGO stimuli (all Tukey tests: $p$ s $<.01$ ). There was also a significant two-way interaction between age and stimulus type. A simple main effect test on this interaction showed that older adults exhibited larger P3 amplitudes than did young adults for irNOGO, but not go and cfNOGO, stimuli.

The simple effect tests on the two-way interaction between no-go probability and stimulus type showed that, for the irNOGO and cfNOGO stimuli, the amplitudes were larger in the $20 \%$ than in the $50 \%$ (and also the $80 \%$, for the irNOGO stimuli) no-go probability condition (irNOGO: $20 \%>50 \% \&$ $80 \%$, all Tukey tests: $p \mathrm{~s}<.01$; cfNOGO: $20 \%>50 \%>80 \%$, all Tukey tests: $p \mathrm{~s}<.01)$.

Summary of P3 data To summarize, the significant main effect of age on P3 peak latencies and amplitudes occurred 
mainly for the irNOGO stimuli, but not the cfNOGO stimuli-that is, the $\mathrm{P} 3$ peak latency occurred later and the $\mathrm{P} 3$ peak amplitude was larger for the older than for the younger adults, but only on irNOGO trials (Table 2). In addition, for both age groups, the $\mathrm{P} 3$ amplitudes for both irNOGO and cfNOGO stimuli were modulated by the no-go probability, with the largest P3 amplitudes being associated with $20 \%$ no-go probability.

\section{Discussion}

In this study, we aimed to examine whether older adults, relative to younger adults, suffered from generic inhibition, selective inhibition, and/or distraction deficits, assessed by behavioral and electrophysiological measures in a go/no-go task paradigm that included manipulations of no-go stimulus type (irrelevant vs. conflict) and probability $(20 \%, 50 \%$, and $80 \%$ no-go stimuli).

\section{Behavioral findings}

The present behavioral results showed that the older adults performed more poorly than the younger adults, as reflected by their slower RTs on go trials, additional omission errors on go trials, and additional commission errors on no-go trials. This effect of age on the behavioral performance of the groups in the go/no-go task paradigm was not accompanied by an interaction with no-go stimulus probability (the degree of go-prepotency), even though we did observe a significant main effect of no-go probability on both RTs and PEs. The effect of age did not interact with stimulus type, in that both age groups committed more errors for cfNOGO than for irNOGO trials, suggesting that it might be more difficult to withhold a response in cfNOGO than in irNOGO trials.

The observed inferior performance of the older adults in a go/no-go task is consistent with the well-known frontal lobe hypothesis (Dempster, 1992; Dennis \& Cabeza, 2008; Raz, 2000; West, 2000). Many studies have reported similar results and suggested generic inhibition deficits in the elderly (Bokura, Yamaguchi, Matsubara, \& Kobayashi, 2002; Lucci, Berchicci, Spinelli, Taddei, \& Di Russo, 2013; Pires et al., 2014; Vallesi, 2011; Vallesi, Stuss, McIntosh, \& Picton, 2009); however, relatively few studies have investigated age-related differences in the processing of different types of no-go stimuli (but see Vallesi et al., 2009) or of different contexts of go/no-go stimulus probabilities. In our previous series of studies using flanker task paradigms, we observed that the ability of older adults to overcome flanker interference was modulated by either different degrees of response bias (Hsieh \& Fang, 2012) or different types of central targets (Hsieh \& Lin, 2014). It is thus of empirical and theoretical interest to determine whether the ability of older adults to suppress prepotent responses can also be modulated by some task parameters. Although we observed that the no-go probability and no-go trial type modulated the participants' performance in the go/no-go task paradigm, these factors did not further interact with age-related differences in go/no-go behavioral performance (i.e., the older adults exhibited slower go RTs, higher go omission error rates, and higher no-go commission error rates). Hence, the behavioral results seem to suggest a generic inhibition deficit in older adults. Yet, because overt responses cannot be directly measured for no-go trials, and therefore inhibitory function can be inferred only through the observation of indirect commission errors, it is unclear whether the underlying processes that are sensitive to aging are similar across the different types of no-go stimuli and/or the different no-go probability conditions. Hence, in the present study we adopted an electrophysiological approach to uncover the neural processes underlying the age-related differences in suppression during no-go trials. We computed the P3 and N2 components because the functions of these components are closely related to inhibition and/or response conflict monitoring.

\section{ERP findings}

The present ERP results generally echo the behavioral findings, but they also provide additional information regarding the processing of no-go stimuli. Regarding the main effect of aging, whereas the behavioral data show inferior performance by the elderly as compared to young adults, the N2 peak latency occurred later and the N2 peak amplitude was smaller for the elderly. The smaller N2 associated with more errors has often been presented as evidence for reduced inhibition efficacy (Roche et al., 2005; van Boxtel et al., 2001) and/or inefficient conflict monitoring (Nieuwenhuis et al., 2003; van Veen \& Carter, 2002a, b). Hence, the present ERP data seem to be in line with the behavioral data, suggesting either a generic age-related inhibition deficit or an age-related conflict-monitoring deficit.

The present $\mathrm{N} 2$ and $\mathrm{P} 3$ data provide additional information to dissociate the mechanisms for processing different types of no-go stimuli that could not be provided by the behavioral data. That is, although the behavioral data showed no significant interaction of age and stimulus type, the N2 and P3 peak amplitudes did show such an interaction. Furthermore, these interactions on the N2 and $\mathrm{P} 3$ peak amplitudes were dissociable - that is, whereas the older adults exhibited larger P3 peak amplitudes and delayed $\mathrm{P} 3$ peak latencies specifically for irNOGO but not for cfNOGO trials relative to the younger adults, they exhibited smaller N2 amplitudes specifically for cfNOGO trials. The results suggest that irNOGO and cfNOGO stimuli might elicit different processes that are differentially impacted by aging as compared to the go stimuli, the irNOGO stimuli were more easily distinguished because 
the difference was in the semantic category of the stimuli (i.e., numbers vs. letters), whereas the cfNOGO stimuli were not easily distinguished because the difference was related to the junction between features (i.e., red $\mathrm{O} / \mathrm{blue} \mathrm{X}$ vs. blue $\mathrm{O} / \mathrm{red}$ $\mathrm{X})$. Therefore, the increased P3 amplitude and earlier P3 latency for irNOGO trials seems to suggest that the older adults were less able to overcome the distractions and processed the irrelevant visual information more than was necessary. Using the oddball paradigm, Sawaki and Katayama (2008) suggested that rare distractors might capture attention, which enables attention to be allocated to the irrelevant information (similar to the irNOGO stimuli used in this study). Therefore, the age-related differences in irNOGO performance PEs might be related to the reduced ability of the older adults to overcome distraction. Conversely, when elicited by cfNOGO stimuli, errors would probably more directly involve conflict-monitoring processes (Smith et al., 2008; Vallesi et al., 2009). Accordingly, it is not surprising to observe a significant main effect of age on N2 amplitudes specifically for cfNOGO stimuli. Therefore, the age-related differences in cfNOGO performance PEs might be related to the reduced ability of older adults to inhibit response prepotency under conditions in which both go and cfNOGO stimuli share similar features, which elicits higher conflict. The present findings of the seesaw functions between the N2 and P3 are consistent with some previous studies suggesting that the N2 primarily reflects conflict-related effects, whereas the $\mathrm{P} 3$ predominately reflects motor inhibition (Enriquez-Geppert et al., 2010; Huster et al., 2013).

With regard to the manipulation of no-go stimulus probability, the behavioral data showed a significant interaction of no-go probability and stimulus type, and the present ERP results also showed similar interactions for both the N2 and P3 peak amplitudes. In addition, such an interaction was not further modulated by aging; that is, both age groups showed that the amplitudes of the $\mathrm{N} 2$ and $\mathrm{P} 3$ varied significantly as a function of the no-go probability, with larger amplitudes being associated with the $20 \%$ no-go probability rather than the other probability conditions. Hence, the present behavioral and ERP results consistently showed age-related declines in go/no-go task performance, regardless of no-go probability and the type of no-go stimulus. When we began this study, the $20 \%$ no-go probability was expected to elicit maximal engagement of executive control to inhibit no-go stimuli (Bruin \& Wijers, 2002; Ford et al., 2004); hence, one would further expect to observe a more pronounced effect of age in the $20 \%$ no-go probability condition, because the elderly are known to suffer from frontal lobe decline (Dempster, 1992; Dennis \& Cabeza, 2008; Raz, 2000; West, 2000). One possibility for why we did not observe the interaction of no-go probability with age-related performance may be due to our between-subjects design. Nevertheless, this speculation cannot fully explain why we could still successfully induce the degree of control demand, as reflected in RTs and PEs, even though such a factor did not interact with age-related performance. Another possibility for not finding age effects on performance at different no-go probabilities may be low power due to the small number of trials split across the multiple conditions. Future study will be needed to clarify these possibilities.

\section{Theoretical implications}

In this study we set out to test some well-known age-related hypotheses: the generic-inhibition-deficit (Hasher \& Zacks, 1988), the selective-inhibition-deficit (Anguera \& Gazzaley, 2012), and the distractibility theories of aging (Healey et al., 2008). The present results, showing dissociable age-related ERP modulations for cfNOGO and irNOGO stimuli, as reflected in the $\mathrm{N} 2$ and $\mathrm{P} 3$, respectively, appeared to support both the selective-inhibition-deficit and distractibility theories of aging. Some previous studies using either the same go/nogo task or other inhibition-related tasks have also suggested selective age-related inhibition deficits (e.g., Anguera \& Gazzaley, 2012; Falkenstein et al., 2002; Hsieh \& Lin, 2014; Kramer et al., 1994). On the other hand, other studies have demonstrated that older adults' performance can be disrupted by distraction, hence supporting the distractibility theory of aging (see Healey et al., 2008, for a review). Therefore, the major contribution of the present study is to provide evidence showing that both hypotheses are not exclusive in describing the age-related deficits in performing a go/no-go task.

In addition, the present findings of dissociable age-related modulations on the N2 and P3 components, evoked by cfNOGO and irNOGO stimuli, respectively, also provide theoretical implications regarding the functional meanings of the N2 and P3: The N2 seemed to be more sensitive to conflict information, whereas the $\mathrm{P} 3$ was more related to inhibition of distraction.

\section{Conclusion}

To conclude, this study has provided information showing that the inhibition deficits of older adults in a go/no-go task paradigm might be due to different underlying mechanisms: disproportionate processing of irrelevant no-go stimuli when the stimuli are easily distinguishable from the target stimuli, and disproportionate suppression of conflicting information when executing or withholding a response when the no-go stimuli share similar features with the go stimuli.

Author note We thank the Ministry of Science Technology of the Republic of China, Taiwan, for financially supporting this research (Contract No. 101-2410-H-006-046-MY3). In addition, this research was, in part, supported by the Ministry of Education, Taiwan, R.O.C., through a grant 
from the Aim for the Top University Project to the National Cheng Kung University. We also thank the American Manuscript Editors company (www.americanmanuscripteditors.com) for English proofreading.

\section{References}

Anguera, J. A., \& Gazzaley, A. (2012). Dissociation of motor and sensory inhibition processes in normal aging. Clinical Neurophysiology, 123, 730-740.

Barkley, R. A. (1997). Behavioral inhibition, sustained attention, and executive functions: constructing a unifying theory of ADHD. Psychological Bulletin, 121, 65-94.

Bokura, H., Yamaguchi, S., Matsubara, M., \& Kobayashi, S. (2002). Frontal lobe contribution to response inhibition process-An ERP study and aging effect. International Congress Series, 1232, 17-20.

Bruin, K., \& Wijers, A. (2002). Inhibition, response mode, and stimulus probability: A comparative event-related potential study. Clinical Neurophysiology, 113, 1172-1182.

Carlson, M. C., Hasher, L., Zacks, R. T., \& Connelly, S. L. (1995). Aging, distraction, and the benefits of predictable location. Psychology and Aging, 10, 427-436.

Dempster, F. N. (1992). The rise and fall of the inhibitory mechanism: Toward a unified theory of cognitive development and aging. Developmental Review, 12, 45-75.

Dennis, N. A., \& Cabeza, R. (2008). Neuroimaging of healthy cognitive aging. In F. I. M. Craik \& T. A. Salthouse (Eds.), Handbook of aging and cognition (3rd ed., pp. 1-54). Mahwah, NJ: Erlbaum.

Donchin, E., \& Coles, M. G. H. (1988). Is the P300 component a manifestation of context updating? Behavioral and Brain Sciences, 11, 357-374. doi:10.1017/S0140525X00058027. disc. 374-427.

Enriquez-Geppert, S., Konrad, C., Pantev, C., \& Huster, R. J. (2010). Conflict and inhibition differentially affect the N200/P300 complex in a combined go/nogo and stop-signal task. NeuroImage, 51, 877887. doi:10.1016/j.neuroimage.2010.02.043

Eriksen, B. A., \& Eriksen, C. W. (1974). Effects of noise letters upon the identification of a target letter in a nonsearch task. Perception \& Psychophysics, 16, 143-149. doi:10.3758/BF03203267

Falkenstein, M., Hoormann, J., \& Hohnsbein, J. (1999). ERP components in go/nogo tasks and their relation to inhibition. Acta Psychologica, 101, 267-291.

Falkenstein, M., Hoormann, J., \& Hohnsbein, J. (2002). Inhibitionrelated ERP components: Variation with modality, age, and timeon-task. Journal of Psychophysiology, 16, 167.

Fallgatter, A. J., \& Strik, W. K. (1999). The nogo-anteriorization as a neurophysiological standard-index for cognitive response control. International Journal of Psychophysiology, 32, 233-238.

Folstein, M. F., Folstein, S. E., \& McHugh, P. R. (1975). "Mini Mental State": A practical method for grading the cognitive state of patients for the clinician. Journal of Psychiatric Research, 12, 189-198. doi: 10.1016/0022-3956(75)90026-6

Ford, J. M., Gray, M., Whitfield, S. L., Turken, U., Glover, G., Faustman, W. O., \& Mathalon, D. H. (2004). Acquiring and inhibiting prepotent responses in schizophrenia: Event-related brain potentials and functional magnetic resonanceimaging. Archives of General Psychiatry, 61, 119-129.

Garavan, H., Ross, T., \& Stein, E. (1999). Right hemispheric dominance of inhibitory control: An event-related functional MRI study. Proceedings of the National Academy of Sciences, 96, 8301-8306.

Gazzaley, A., \& D'Esposito, M. (2007). Top-down modulation and normal aging. Annals of the New York Academy of Sciences, 1097, 6783. doi:10.1196/annals.1379.010

Hasher, L., \& Zacks, R. T. (1988). Working memory, comprehension, and aging: A review and a new view. In G. H. Bower (Ed.), The psychology of learning and motivation: Advances in research and theory (Vol. 22, pp. 193-225). San Diego, CA: Academic Press. doi: 10.1016/S0079-7421(08)60041-9

Hasher, L., Zacks, R. T., \& May, C. P. (1999). Inhibitory control, circadian arousal, and age. In D. Gopher \& A. Koriat (Eds.), Attention and performance XVII: Cognitive regulation of performance. Interaction of theory and application (pp. 653-675). Cambridge, MA: MIT Press.

Healey, M. K., Campbell, K. L., \& Hasher, L. (2008). Cognitive aging and increased distractibility: Costs and potential benefits. Progress in Brain Research, 169, 353-363.

Hommel, B., Li, K. Z., \& Li, S.-C. (2004). Visual search across the life span. Developmental Psychology, 40, 545-558.

Hsieh, S., \& Fang, W. (2012). Elderly adults through compensatory responses can be just as capable as young adults in inhibiting the flanker influence. Biological Psychology, 90, 113-126.

Hsieh, S., Liang, Y.-C., \& Tsai, Y.-C. (2012). Do age-related changes contribute to the flanker effect? Clinical Neurophysiology, 123, 960-972.

Hsieh, S., \& Lin, Y.-C. (2014). The boundary condition for observing compensatory responses by the elderly in a flanker-task paradigm. Biological Psychology, 103, 69-82.

Huster, R. J., Enriquez-Geppert, S., Lavallee, C. F., Falkenstein, M., \& Herrmann, C. S. (2013). Electroencephalography of response inhibition tasks: Functional networks and cognitive contributions. International Journal of Psychophysiology, 87, 217-233.

Kok, A. (1999). Varieties of inhibition: manifestations in cognition, event-related potentials and aging. Acta Psychologica, 101, 129158.

Kramer, A. F., Hahn, S., \& Gopher, D. (1999). Task coordination and aging: Explorations of executive control processes in the task switching paradigm. Acta Psychologica, 101, 339-378.

Kramer, A. F., Humphrey, D. G., Larish, J. F., \& Logan, G. D. (1994). Aging and inhibition: Beyond a unitary view of inhibitory processing in attention. Psychology and Aging, 9, 491-512. doi:10.1037/ 0882-7974.9.4.491

Lucci, G., Berchicci, M., Spinelli, D., Taddei, F., \& Di Russo, F. (2013). The effects of aging on conflict detection. PLoS ONE, 8, e56566. doi:10.1371/journal.pone.0056566

MacDonald, A. W., Cohen, J. D., Stenger, V. A., \& Carter, C. S. (2000). Dissociating the role of the dorsolateral prefrontal and anterior cingulate cortex in cognitive control. Science, 288, 1835-1838. doi:10. 1126/science.288.5472.1835

Mayr, U. (2001). Age differences in the selection of mental sets: The role of inhibition, stimulus ambiguity, and response-set overlap. Psychology and Aging, 16, 96-109.

Nieuwenhuis, S., Yeung, N., van den Wildenberg, W., \& Ridderinkhof, K. R. (2003). Electrophysiological correlates of anterior cingulate function in a go/no-go task: Effects of response conflict and trial type frequency. Cognitive, Affective, \& Behavioral Neuroscience, 3, 17-26. doi:10.3758/CABN.3.1.17

Pires, L., Leitão, J., Guerrini, C., \& Simões, M. R. (2014). Event-related brain potentials in the study of inhibition: Cognitive control, source localization and age-related modulations. Neuropsychology Review, 24, 461-490. doi:10.1007/s11065-014-9275-4

Raz, N. (2000). Aging of the brain and its impact on cognitive performance: Integration of structural and functional findings. In F. I. M. Craik \& T. A. Salthouse (Eds.), Handbook of aging and cognition (2nd ed., pp. 1-90). Mahwah, NJ: Erlbaum.

Roberts, L. E., Rau, H., Lutzenberger, W., \& Birbaumer, N. (1994) Mapping P300 waves onto inhibition: Go/no go discrimination. Electroencephalography and Clinical Neurophysiology, 92, 44-55.

Roche, R. A., Garavan, H., Foxe, J. J., \& O'Mara, S. M. (2005). Individual differences discriminate event-related potentials but not performance during response inhibition. Experimental Brain Research, 160, 60-70. 
Sawaki, R., \& Katayama, J. I. (2008). Distractor P3 is associated with attentional capture by stimulus deviance. Clinical Neurophysiology, 119, 1300-1309.

Scialfa, C. T., Esau, S. P., \& Joffe, K. M. (1998). Age, target-distractor similarity, and visual search. Experimental Aging Research, 24, 337-358.

Smith, J. L., Johnstone, S. J., \& Barry, R. J. (2007). Response priming in the Go/NoGo task: The N2 reflects neither inhibition nor conflict. Clinical Neurophysiology, 118, 343-355.

Smith, J. L., Johnstone, S. J., \& Barry, R. J. (2008). Movement-related potentials in the Go/NoGo task: The $\mathrm{P} 3$ reflects both cognitive and motor inhibition. Clinical Neurophysiology, 119, 704-714.

Tekok-Kilic, A., Shucard, J. L., \& Shucard, D. W. (2001). Stimulus modality and Go/NoGo effects on P3 during parallel visual and auditory continuous performance tasks. Psychophysiology, 38, 578-589.

Vallesi, A. (2011). Targets and non-targets in the aging brain: A go/nogo event-related potential study. Neuroscience Letters, 487, 313-317. doi:10.1016/j.neulet.2010.10.046
Vallesi, A., Stuss, D. T., McIntosh, A. R., \& Picton, T. W. (2009). Agerelated differences in processing irrelevant information: Evidence from event-related potentials. Neuropsychologia, 47, 577-586. doi:10.1016/j.neuropsychologia.2008.10.018

van Boxtel, G. J., van der Molen, M. W., Jennings, J. R., \& Brunia, C. H. (2001). A psychophysiological analysis of inhibitory motor control in the stop-signal paradigm. Biological Psychology, 58, 229-262.

van Veen, V., \& Carter, C. S. (2002a). The anterior cingulate as a conflict monitor: fMRI and ERP studies. Physiology and Behavior, 77, 477482.

van Veen, V., \& Carter, C. S. (2002b). The timing of actionmonitoring processes in the anterior cingulate cortex. Journal of Cognitive Neuroscience, 14, 593-602. doi:10. $1162 / 08989290260045837$

West, R. (2000). In defense of the frontal lobe hypothesis of cognitive aging. Journal of the International Neuropsychological Society, 6, $727-729$. 